\title{
Thermal exchange of glass micro-fibers measured by the $3 \omega$ technique
}

\author{
T.D. Nguyen \\ Institut NEEL, Univ. Grenoble Alpes \\ CNRS-25 avenue des Martyrs \\ F-38042 Grenoble, France \\ e-mail: tuyenkstnk46@gmail.com
}

\author{
J. Richard \\ Institut NEEL, Univ. Grenoble Alpes \\ CNRS-25 avenue des Martyrs \\ F-38042 Grenoble, France \\ e-mail: jacques.richard@neel.cnrs.fr
}

\author{
J. Doumouro \\ Institut Langevin, ESPCI Paris \\ Université PSL, CNRS, 1 rue Jussieu, \\ F-75005, Paris, France \\ e-mail: joris.doumouro@espci.fr
}

\author{
Y. De Wilde \\ Institut Langevin, ESPCI Paris \\ Université PSL, CNRS, 1 rue Jussieu, \\ F-75005, Paris, France \\ e-mail: yannick.dewilde@espci.fr
}

\author{
O. Bourgeois * \\ Institut NEEL, Univ. Grenoble Alpes \\ CNRS-25 avenue des Martyrs \\ F-38042 Grenoble, France \\ e-mail: olivier.bourgeois@neel.cnrs.fr
}

\begin{abstract}
In this work, we propose an experimental set-up to measure the thermal conductivity and specific heat of a single suspended glass fiber, as well as the thermal contact resistance between two glass fibers. By using optical lithography, wet and dry etching and thin film deposition, we prepared suspended glass fibers that are coated by niobium nitride thin film $(\mathrm{NbN})$ used as room temperature thermal transducer. By using the $3 \omega$ technique, the thermal conductivity of glass fiber was measured to be $1.1 \mathrm{~W} \cdot \mathrm{m}^{-1} \cdot \mathrm{K}^{-1}$ and specific heat $0.79 \mathrm{~J} \cdot \mathrm{g}^{-1} \cdot \mathrm{K}^{-1}$ around $300 \mathrm{~K}$ under vacuum conditions. By introducing exchange gas into the measurement chamber, influence of the gas on the heat transfer was studied, and the convection coefficient $h$ for all the measurement ranges from a pressure of $0.01 \mathrm{hPa}$ to $1000 \mathrm{hPa}$, over more than five orders of magnitude, has been obtained. By adding a bridging glass fiber on top of two other suspended glass fibers, it was possible to estimate the thermal contact resistance between two glass fibers $R_{c}$ in the range of $10^{7} \mathrm{~K} . \mathrm{W}^{-1}$ to $10^{8} \mathrm{~K} . \mathrm{W}^{-1}$.
\end{abstract}

\section{Introduction}

Nowadays, the energy loss is a common problem world wild. As an example, a large part of the energy consumption goes to heat buildings $(\approx 40 \%$ in Europe); the heat leaks being due to bad thermal isolation $[1,2]$. One of the solutions for this problem is to reduce the heat loss (by the roof, the walls and the windows) by developing advanced materials, particularly thermal insulation materials for buildings. Most traditional insulation materials have a thermal conductivity between 30 to $50 \mathrm{~mW} \cdot \mathrm{m}^{-1} \cdot \mathrm{K}^{-1}$ at $10^{\circ} \mathrm{C}[3,4]$. Thanks to recent progress in this field, there have been advanced insulation materials developed having thermal conductivity lower than that of traditional insulation material such as aerogel silica showing a thermal conductivity of $18 \mathrm{~mW} \cdot \mathrm{m}^{-1} \cdot \mathrm{K}^{-1}$, or Vacuum Insulation Panels (VIP) based on nanostructured silica or glass fiber with a thermal conductivity as low as $7 \mathrm{~mW} \cdot \mathrm{m}^{-1} \cdot \mathrm{K}^{-1}$. However, current thermal models of these innovative materials predict much lower thermal conductivity in relative disagreement with the one that is measured at the macroscale.

${ }^{*}$ Corresponding author. 
In order to improve the insulating capacity of the composite materials, one must understand all the mechanisms of the heat transfer through the insulation material including convection, conduction in the solid phases and through the air as well as by radiation. In general, the thermal conductivity characterization of insulation materials is based on a macroscopic sample with a size bigger than $10 \mathrm{~cm}$. This characterization technique is enough to give the effective thermal conductivity of the bulk material. However, to further improve the performance of the insulation materials, one needs to have information on the contribution of each individual heat transfer mechanisms at microscopic scale (conduction, convection, radiation) along with each building blocks. In VIP insulating material, the building block is based on glued single glass fibers.

To characterize the heat conduction in one dimensional systems such as micro/nano fibers, different techniques are available, including $3 \omega$ technique [5-18], optical heating-electrical thermal sensing technique [19], pulsed laserassisted thermal relaxation technique [20,21] and transient electrothermal (TET) technique [22]. Among those techniques, the longitudinal $3 \omega$ method is the most widely used for the measurement of suspended individual $1 \mathrm{D}$ samples $[6,9]$. To implement the $3 \omega$ technique, a transducer deposited on top of the suspended structure will serve as a heater and as a thermometer at the same time. This transducer is connected to a current source which supplies an AC current oscillating at an angular frequency $1 \omega$. This heating current will generate a voltage oscillating with an angular frequency $3 \omega$, which is directly connected to the thermal conductivity and specific heat of the specimen. So by measuring this $3 \omega$ voltage, we will be able to extract the thermal conductivity and specific heat of the sample.

Besides applications in the study of heat conduction in solid materials, the $3 \omega$ technique has been recently applied to study the heat transfer in liquid and gas media as well as developed as a sensor for gas and liquid detection $[18,23,24]$. Most of the previous works are dedicated to the measurement of thermal conductivity of the liquid or sensing the amount of a specific liquid or gas. Recently in 2018, Gao and coworkers have reported on the thermal transport around a micro-wire based on a steady-state hot-wire method [25]. They used a platinum (Pt) wire with a diameter of $25 \mu \mathrm{m}$ acting as thermometer and heater. By using a DC pulse applied to the Pt wire and measuring the temperature of the $\mathrm{Pt}$ wire as function of gas pressure, they were able to calculate the heat convection coefficient at different gas pressure.

Unlike the heat conduction in one dimensional system, the thermal transport at the interface between one dimensional systems is not widely studied yet. In recent years, there have been some experimental works on this problem, for examples, thermal contact resistance between single carbon nanotubes [26], the heat transfer in array of aligned $\mathrm{TiO}_{2}$ nanotubes [27] and the thermal contact resistance between Pt wires [28]. Besides that, the T-type probe technique $[29,30]$ appears to be interesting due to its practicalities. In this technique, a hot wire, which is heated by a current, is in contact with one end of a to-be-measured wire, the other end of the to-be-measured wire is in contact to the heat sink. The properties of the sample can be determined by measuring the temperature change on the hot wire before and after being in contact with the sample. To the best of our knowledge, there are no work on the study of the heat convection and conduction at the micro-scale on glass fibers.

In the present work, we propose an experimental set-up to measure thermal conductivity, specific heat of a single suspended micro glass fiber based on $3 \omega$ technique. And by placing a micro glass fiber on top of two other suspended ones, we can also measure the thermal contact resistance between two glass fibers based on modified transient electrothermal technique [22] or an "H-type probe" 1. By introducing to the measurement chamber an exchange gas, we analyzed the influence of the gas pressure on the heat conduction through the convection mechanism. With the proposed experiment, we are able to access the thermal information of the most important building block of the insulation materials in terms of conduction of the solid phase as well as the influence of the gas due to the convection heat transport.

\section{Methods}

In order to measure the thermal conductivity of a single glass fiber by the $3 \omega$ method, first the glass fiber is suspended between two electrodes, then a niobium nitride $(\mathrm{NbN})$ thermometric thin film is deposited on the glass fiber to serve as transducer in the experiment [31]. To fix the fiber on the electrodes, silver paste is used since it is a good thermal and electrical conductor. To keep the fiber suspended, the substrate underneath in silicon is etched to make the electrodes look like pillars. Since a thermometric layer is deposited on the fiber, the pillar-like electrodes have to have a bottleneck shape to ensure that the transducer is electrically isolated from the ground.

\subsection{Fabrication process}

The fabrication of the sample begins from a Si substrate coated by silicon nitride (SiN) with a size of $1 \mathrm{~cm}^{2}$. By using sputtering, a layer of WTi/Au $(5 \mathrm{~nm} / 100 \mathrm{~nm})$ is deposited on the Si substrate (Fig. 1 a). The WTi/Au

\footnotetext{
${ }^{1} \mathrm{~A}$ glass fiber is put on top of other two glass fibers forming an $\mathrm{H}$ shape.
} 
a

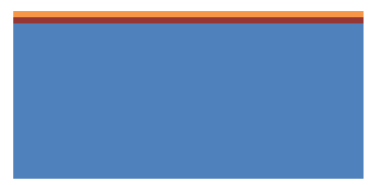

b
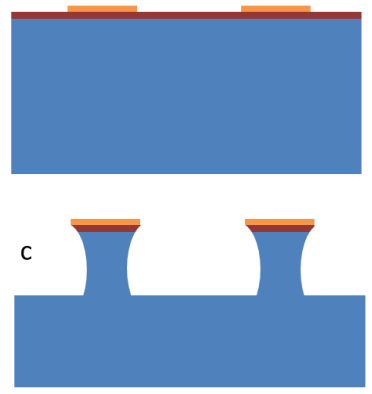

d

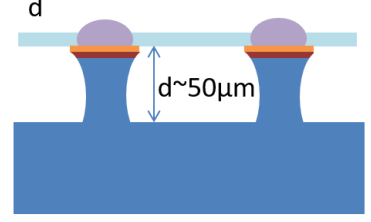

e

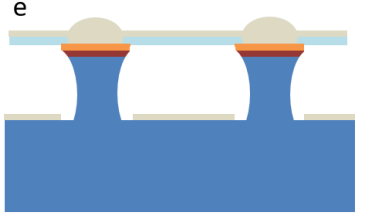

$\begin{array}{ll}\mathrm{NbN} & \mathrm{Si} \\ \mathrm{Au} / \mathrm{WTi} & \mathrm{SiN} \\ & \text { Glass fiber } \\ & \text { Silver paste }\end{array}$

Fig. 1. Micro-fabrication steps for the preparation of suspended glass fibers: a) WTi/Au layer is deposited on the Si substrate, b) Au contacts are structured after the lithography and wet etching, c) contacts in the shape of pillar are obtained after dry $\mathrm{XeF}_{2}$ etching, d) the glass fiber is suspended and installed on the contacts by silver paste, e) the sample is completed after deposition by sputtering of the $\mathrm{NbN}$ thermometer on top of the glass fiber.

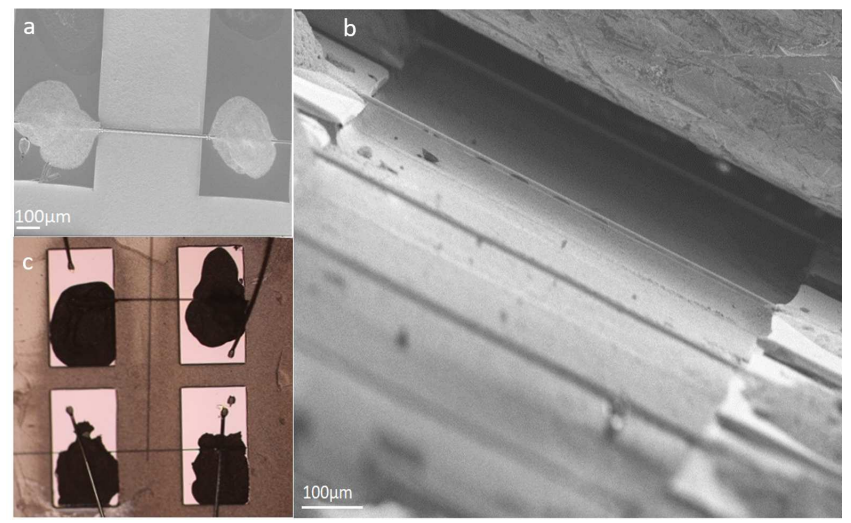

Fig. 2. a) SEM picture of a suspended glass fiber b) SEM picture of the glass fiber obtained under a tilted angle of $90^{\circ}$ showing clearly the suspension between the two electrodes $\mathrm{c}$ ) optical image of the $\mathrm{H}$-type probe geometry for the measurement of thermal contact resistances.

contacts are obtained by lithography and wet etching of WTi/Au by KI and $\mathrm{H}_{2} \mathrm{O}_{2}$ solutions as shown in Fig. 1 b. After removing the photoresist by acetone and ethanol, the sample is etched using $\mathrm{SF}_{6}$ to remove the SiN layer, then loaded immediately into a chamber for vapor etching $\mathrm{Si}$ using $\mathrm{XeF}_{2}$ gas. The step of etching $\mathrm{Si}$ by $\mathrm{XeF}_{2}$ is to obtain contacts with bottleneck shape as shown in Fig. 1 c.

The glass fiber ${ }^{2}$ is then placed on top of the contacts and fixed by drops of silver paste as shown in Fig. 1 d. Finally, a $140 \mathrm{~nm}$ thick film of NbN thermometer/heater is deposited on the fiber by pulsed DC sputtering. Since the contacts have a shape of bottleneck, the thermometer deposited on the fiber is electrically isolated from the ground as shown in Fig. 1 e. Figure 2 a shows the suspended glass fiber, visualized by Scanning Electron Microscopy (SEM). By tilting the sample at an angle of $90^{\circ}$, the fiber is observed under SEM as shown in Fig. 2 b. From this picture, we clearly see that the fiber is well suspended and separated from the Si substrate.

Niobium nitride is selected for heater/thermometer in this experiment because it is a well known MottAnderson insulator with an increasing resistance as the temperature is decreased [31]. NbN has better sensitivity than other metallic thermometer even at room temperature; indeed the temperature coefficient of resistance (TCR) of $\mathrm{NbN}$ at $300 \mathrm{~K}$ is about $6 \times 10^{-3}-7 \times 10^{-3} \mathrm{~K}^{-1}$ in comparison to $2 \times 10^{-3} \mathrm{~K}^{-1}$ of Pt thin film thermometer. Moreover, $\mathrm{NbN}$ has a much lower electrical conductivity than that of regular metallic thermometers. As a consequence, by using the Wiedemann-Franz law, we can deduce that its thermal conductivity is really low and so this thermometry is perfectly suitable for thermal measurements of low thermal conductivity materials like glass fiber in the geometry of longitudinal $3 \omega$ method.

\footnotetext{
${ }^{2}$ The glass fibers are standard isolation glass supplied by Saint-Gobain Research (SGR).
} 


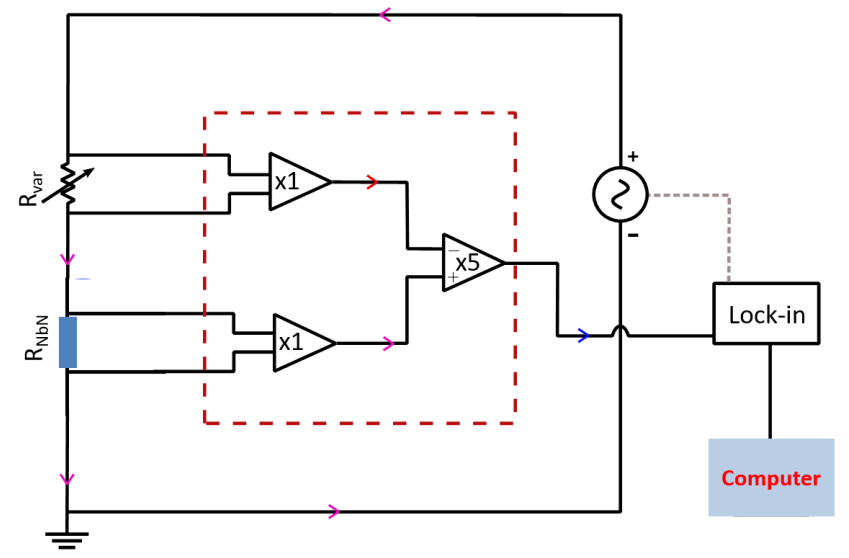

Fig. 3. The electronic set-up dedicated to the $3 \omega$ technique is based on a differential bridge. The voltage signal on the thermometer is compared to the voltage on the variable resistor $R_{v a r}$ in order to extract their difference. By this operation, the dominating $1 \omega$ voltage is minimized, and hence the $3 \omega$ signal can be extracted with the smallest noise when the variable resistor is set to have the same resistance as the thermometer.

\subsection{Experimental protocol of the $3 \omega$ measurement techniques}

The suspended fiber is heated by an AC current with frequency $1 \omega, I=I_{0} \sin (\omega t)$. Due to Joule heating power $P=P_{d c}+P_{a c} \cos (2 \omega t)$, the temperature of the transducer increases and oscillates at the angular frequency $2 \omega, T=$ $T_{d c}+T_{a c} \cos (2 \omega t+\varphi)$, leading to the oscillation of the resistance of the thermometer at angular frequency $2 \omega, R_{t h}=$ $R_{0}\left(1+\alpha_{T C R} T_{d c}+\alpha_{T C R} T_{a c} \cos (2 \omega t+\varphi)\right)$, where $\alpha_{T C R}$ is the TCR of the transducer. Therefore the voltage generated by the oscillation of the temperature will oscillate at angular frequency $3 \omega, V=I_{0} R_{0}\left[A \sin (\omega t)+\alpha_{T C R} T_{a c} \sin (3 \omega t+\phi)\right]$. Since the amplitude of the temperature oscillation $T_{a c}$ is sensitive to both the thermal conductivity and the specific heat of the fiber, by measuring the $3 \omega$ voltage, one is able to extract the thermal conductivity and the specific heat of the suspended fiber.

In the experiment, in order to determine the thermal conductivity $k$ and the specific heat $c_{p}$ of the sample, we made the measurements of $V_{3 \omega}$ versus frequency under high vacuum for different samples with different lengths. In a second step, a similar measurement will be performed with the chamber filled with $\mathrm{N}_{2}$ gas. Under controlled pressure of the gas, a set of $V_{3 \omega}$ measurements are made for different pressures. By fitting $V_{3 \omega}$ with a mathematical model that will be developed in the following, knowing the value of $k$ and $c_{p}$ from the measurements under vacuum, the convective heat transfer coefficient between the fiber and the rest of the experimental chamber will be extracted as a function of gas pressure.

\subsection{Electronic set-up and thermometer calibration}

The fabricated device dedicated to the $3 \omega$ technique (as shown in Fig. 2) is mounted on the sample holder loaded into the measurement chamber and pumped to a high vacuum $\left(p \sim 5 \times 10^{-4} \mathrm{hPa}\right)$. In order to have the best sensitivity on the $3 \omega$ signal, the thermometer of the fiber is measured using a differential bridge circuit as shown in Fig. 3. In this circuit the NbN thermometer (on the fiber) is connected in series to a variable resistor and connected to a low noise current source. The voltage signals on the $\mathrm{NbN}$ thermometer and the variable resistor are extracted from the circuit by comparators in the pre-amplifier. Then this voltage difference is monitored by a lock-in amplifier. Before measuring the $3 \omega$ signal, effect from $1 \omega$ voltage is canceled by setting the value of the variable resistor to have the minimum $1 \omega$ voltage at the output of the pre-amplifier.

For each sample, the resistance of $\mathrm{NbN}$ thermometer is first calibrated under high vacuum to obtain the $\mathrm{NbN}$ resistance as a function of temperature. Fig. 4 shows the calibration data of the NbN thermometer on the suspended glass fiber. The red square shows the resistance of the thermometer measured with a four point configuration, and the black square shows the data measured with a two point configuration which is easier to implement. The first important point from this data is that the deposited $\mathrm{NbN}$ has the expected behaviour since the resistance increases as the temperature decreases. The temperature coefficient of resistance is estimated to be about $6 \times 10^{-3} \mathrm{~K}^{-1}$ at room temperature. The second point is that the difference between the two measurement configurations is very small, less than $2 \%$ of the absolute value of the resistance. This means that the electrical contact resistance is small; moreover that resistance is thermally coupled to the heat bath, so we do not expect any spurious $3 \omega$ signal coming from the contact itself. Hence two point or four point configuration will not make a significant difference. Regarding thermal transport in the thermometer itself, by using the Wiedemann-Franz law, we can estimate the thermal conductance of the $\mathrm{NbN}$ layer to be of the order of $10^{-10} \mathrm{~W} . \mathrm{K}^{-1}$, two orders of 


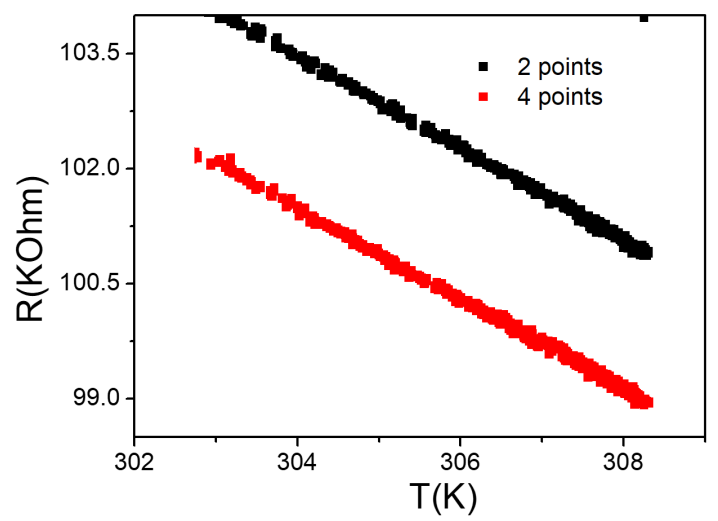

Fig. 4. Calibration of the $\mathrm{NbN}$ thermometer deposited on suspended glass fiber. To avoid overheating, an $\mathrm{AC}$ current of $100 \mathrm{nA}$ is applied to the thermometer, the calibration is done by measuring the voltage using a lock-in amplifier at different temperatures.

magnitude less that any thermal conductance of glass fibers measured in this work.

3 Mathematical model describing the temperature variation of the suspended fiber

3.1 Solving the 3D heat diffusion differential equation

In the case of suspended glass fiber, the filament can be approximated by a glass rectangular parallelepiped coated by a layer of NbN shown in Fig. 5. This glass parallelepiped is placed at a distance $d$ from the thermal bath at the bottom. The temperature of the thermal bath is set constant at $T_{0}$. In order to have the same density of power distributed in the fiber of diameter $D_{1}$, as the heater is evaporated on a surface $L \pi D_{1} / 2$, we take $2 a=\pi D_{1} / 2$ and $b=D_{1} / 2$, then the surface of the normal section of the fiber is the same as in the real cylindrical glass fiber; here we have investigated glass fibers with a typical diameter $D_{1}=9 \mu \mathrm{m}$, which yields $a=7 \mu \mathrm{m}$ and $b=4.5 \mu \mathrm{m}$.

The temperature along the fiber $T(x, y, z, t)$ is given by the solution to the $3 \mathrm{D}$ heat diffusion differential equation [32]:

$$
\frac{\partial^{2} T}{\partial x^{2}}(x, y, z, t)+\frac{\partial^{2} T}{\partial y^{2}}(x, y, z, t)+\frac{\partial^{2} T}{\partial z^{2}}(x, y, z, t)=\frac{1}{D_{d i f f}} \frac{\partial T}{\partial t}(x, y, z, t)
$$

with $D_{\text {diff }}=\frac{k}{\rho c_{p}}$ is the diffusivity of the fiber, $k$ is the thermal conductivity, $c_{p}$ is specific heat and $\rho$ is the mass density of the fiber. In our experimental condition, the radiation heat loss is neglected.

In order to calculate the solution of Eqn. 1, we need initial and boundary conditions. Due to the symmetry of the problem we only calculate $T$ for $x \geq 0$. The initial condition is : $t=0, T(x, y, z, 0)=T_{0}$ and the boundary conditions are:

$$
\begin{cases}y=0, & T(x, 0, z, t)=T_{0} ; \quad y=L, \quad T(x, L, z, t)=T_{0} \\ x=0, & \frac{\partial T}{\partial x}(0, y, z, t)=0 ; \quad x=a, \quad-k \frac{\partial T}{\partial x}(a, y, z, t)=h_{2 r}\left(T(a, y, z, t)-T_{0}\right) \\ z=0, & \int_{0}^{a} \int_{0}^{L}-k \frac{\partial T}{\partial z}(x, y, 0, t) \mathrm{d} x \mathrm{~d} y=\frac{P_{0}}{4}(1-\cos 2 \omega t)-\int_{0}^{L} \int_{0}^{L} h_{2 r}\left[T(x, y, 0, t)-T_{0}\right] \mathrm{d} x \mathrm{~d} y \\ z=b, & -k \frac{\partial T}{\partial z}(x, y, b)=h_{1 r}\left(T(x, y, b, t)-T_{0}\right)\end{cases}
$$

with the convection heat transport coefficients $h_{1 r}$ for the bottom surface of the fiber and $h_{2 r}$ for the other surfaces of the fiber in the rectangular approximation as shown in Fig. 5 where the subscript $r$ stands for rectangular; in vacuum condition $h_{2 r}=h_{1 r}=0$. The total electrical power through the heater is $P(t)=R_{0} I_{0}^{2} \sin ^{2} \omega t$ or $P(t)=$ $\frac{P_{0}}{2}(1-\cos 2 \omega t)$ with $P_{0}=R_{0} I_{0}^{2} ; R_{0}$ the resistance of the heater at $T=T_{0}$ measured in four probe configuration. Here, we used $\frac{P(t)}{2}$ for the heating power, due to the symmetry of the problem. With the small thickness of the $\mathrm{NbN}$ layer, we neglected the mass of the heater in the solution of Eqn. 1. 
Our model can account for the fact that the sample is placed closed to the bottom of the vacuum chamber $(10 \mu m \leq d \leq 100 \mu m)$ and the convective coefficients are different for the bottom face at $z=b$ and the other faces $\left(h_{1 r} \neq h_{2 r}\right)$. The solution for $T$ is then given by:

$$
T(x, y, z, t)=T_{0}+T_{\text {stat }}(x, y, z)+U(x, y, z, t)+U_{h}(x, y, z, t)
$$

with $T_{\text {stat }}(x, y, z)$ is the stationary part, $U(x, y, z, t)$ is the transient part and $U_{h}(x, y, z, t)$ is the periodic part. At the angular frequency $2 \omega$ only the determination of $U_{h}(x, y, z, t)=U_{h}(x, y, z) e^{2 i \omega t}$ is of importance. By using the Fourier method of separation of variables [32], one obtains for $U_{h}(x, y, z)$ :

$$
U_{h}(x, y, z)=-\sum_{n=1}^{\infty} \sum_{m=0}^{\infty} \sin \left(\lambda_{m} a\right) \frac{P_{0}\left[(-1)^{n}-1\right]}{a L n \pi} \frac{\sin \left(\gamma_{m} y\right) \cos \left(\lambda_{m} x\right)\left[\cosh \left(\alpha_{n m} z\right)-B_{n m} \sinh \left(\alpha_{n m} z\right)\right]}{\lambda_{m} a\left[1+\frac{\sin \left(2 \lambda_{m} a\right)}{2 \lambda_{m} a}\right]\left[h_{2 r}+k \alpha_{n m} B_{n m}\right]}
$$

with $\alpha_{n m}=\left(\gamma_{n}^{2}+\lambda_{m}^{2}+\frac{2 i \omega}{D_{d i f f}}\right)^{1 / 2}$, where $\gamma_{n}=\frac{n \pi}{L}$ and $\lambda_{m}$ are solution of $\tan \left(\lambda_{m} a\right)=h_{2 r} a /\left(\lambda_{m} a k\right)$ and

$$
B_{n m}=\left(1+\frac{\alpha_{n m} k}{h_{1} r} \tanh \left(\alpha_{n m} b\right)\right) /\left(\frac{\alpha_{n m} k}{h_{1 r}}+\tanh \left(\alpha_{n m} b\right)\right)
$$

The mean averaged temperature on $x$ and $y$ at $z=0$ is:

$$
\bar{U}_{h}(z=0)=\sum_{n=1}^{\infty} \sum_{m=0}^{\infty} \frac{P_{0}\left[(-1)^{n}-1\right]^{2}}{a L n^{2} \pi^{2}} \frac{\sin ^{2}\left(\lambda_{m} a\right)}{\left(\lambda_{m} a\right)^{2}} \times \frac{1}{\left[1+\frac{\sin \left(2 \lambda_{m} a\right)}{2 \lambda_{m} a}\right]\left[h_{2 r}+k \alpha_{n m} B_{n m}\right]}
$$

In the real space, the mean temperature is obtained from the following three equations:

$$
\begin{gathered}
U_{r}(z=0)=\operatorname{Re}\left[\bar{U}_{h}(x, y, 0) \times e^{2 i \omega t}\right]=\left[T_{\text {real }} \cos (2 \omega t)-T_{\text {im }} \sin (2 \omega t)\right] \frac{P_{0}}{a L \pi^{2}} \\
T_{\text {real }}=\operatorname{Re} \sum_{n=1}^{\infty} \sum_{m=0}^{\infty} \frac{\left[(-1)^{n}-1\right]^{2}}{n^{2}} \frac{\sin ^{2}\left(\lambda_{m} a\right)}{\left(\lambda_{m} a\right)^{2}\left[1+\frac{\sin \left(2 \lambda_{m} a\right)}{2 \lambda_{m} a}\right]\left[h_{2 r}+k \alpha_{n m} B_{n m}\right]} \\
T_{\text {im }}=\operatorname{Im} \sum_{n=1}^{\infty} \sum_{m=0}^{\infty} \frac{\left[(-1)^{n}-1\right]^{2}}{n^{2}} \frac{\sin ^{2}\left(\lambda_{m} a\right)}{\left(\lambda_{m} a\right)^{2}\left[1+\frac{\sin ^{2}\left(2 \lambda_{m} a\right)}{2 \lambda_{m} a}\right]\left[h_{2 r}+k \alpha_{n m} B_{n m}\right]}
\end{gathered}
$$

Coming back to the $3 \omega$ method, the overall voltage is measured at the two extremities of the filament:

$$
V(t)=\left(R_{0}+\delta R\right) I_{0} \sin (\omega t)
$$

where $\delta R=\left(\frac{d R}{d T}\right)_{T=T_{0}} \times U_{r}$. At the $3 \omega$ angular frequency, the voltage $V_{3 \omega}$ will be related to the temperature oscillation by the relation:

$$
V_{3 \omega}=\frac{1}{2} \frac{R_{0} I_{0}^{3}}{a L \pi^{2}}\left(T_{i m} \cos (3 \omega t)+T_{\text {real }} \sin (3 \omega t)\right)\left(\frac{d R}{d T}\right)_{T=T_{0}}
$$




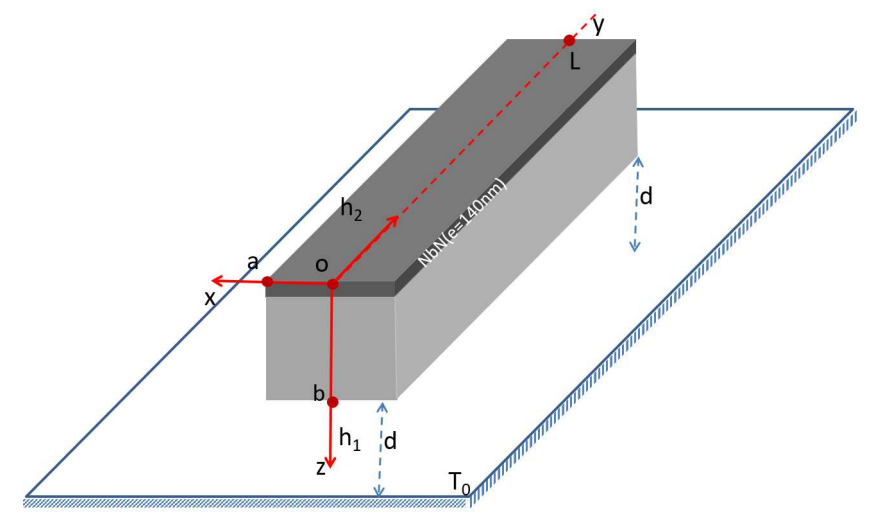

Fig. 5. Model of the glass fiber coated by $\mathrm{NbN}$ (of thickness $e=140 \mathrm{~nm}$ ) as a rectangular parallelepiped placed at a distance $d$ from the thermal bath at the bottom, with the convective heat transport coefficients $h_{1 r}$ for the bottom surface and $h_{2 r}$ for the top and side surfaces, $L$ being the length of the fiber, $b$ the thickness and $a$ the half width of the rectangular parallelepiped.

$$
V_{3 \omega}=\frac{1}{2} \frac{R_{0} I_{0}^{3}}{a L \pi^{2}}\left(\frac{d R}{d T}\right)_{T=T_{0}}\left(T_{\text {real }}^{2}+T_{i m}^{2}\right)^{1 / 2} \sin (3 \omega t+\phi)
$$

with $\tan \phi=\frac{T_{\text {im }}}{T_{\text {real }}}$.

Experimentally, a root mean square (rms) current is applied and hence the measured modulus of the $V_{3 \omega}$ will be given by:

$$
\left(V_{3 \omega}\right)_{r m s}=\frac{R_{0} I_{r m s}^{3}}{a L \pi^{2}}\left(\frac{d R}{d T}\right)_{T=T_{0}}\left(T_{r e a l}^{2}+T_{i m}^{2}\right)^{1 / 2}
$$

This is the crucial equation that will be used later on to fit experimental data and extract the thermal properties of the suspended fiber. Under high vacuum conditions, $h_{2 r}=h_{1 r}=0$, we obtained the same equations for the phase and the RMS modulus as in Lu's work [6]. Finally, the value of $k$ and $c_{p}$ will be obtained by fitting the experimental data of $\left(V_{3 \omega}\right)_{r m s}$ as a function of the angular frequency using Eqn. 12.

If exchange gas is introduced in the measurement chamber, then we will need the value of $h_{1 r}$ and $h_{2 r}$, keeping the same $k$ and $c_{p}$ as obtained from vacuum conditions, to fit the $\left(V_{3 \omega}\right)_{r m s}$ as a function of the angular frequency $\omega$. For this, we need to have a very clear estimation of $h_{1 r}$ and $h_{2 r}$, if one wants to use Eqn. 12 under gas pressure.

\subsection{Evaluation of the convection heat transport coefficients}

In the previous section, to solve the differential equation of the heat diffusion, the glass fiber was approximated as a rectangular parallelepiped, an approximation that eases to find the solution of the $V_{3 \omega}$, with $h_{1 r}$ and $h_{2 r}$ the coefficients of convection corresponding to different surfaces that need to be calculated precisely.

In the heat transfer theory of rarefied gas at rest, the calculations are made in th symmetric cylindrical geometry for instance to evaluate the convection between a cylindrical fiber of diameter $D_{1}$ at a temperature $T_{1}$ and an external concentric cylinder of diameter $D_{2}$ at a temperature $T_{2}$ (with $D_{2}>D_{1}$ ). However, in our experimental case, the geometry of the glass fiber inside the measurement chamber as depicted in Fig. 6 is neither symmetric nor cylindrical. If the glass fiber used in this work is cylindrical, it is however suspended at a distance $d$ from the flat Si substrate that is connected to the thermal bath with $10 \mu \mathrm{m} \leq d \leq 100 \mu \mathrm{m}$. Therefore, we have neither a total cylindrical symmetry nor a planar symmetry. However, the dimensions of the experimental chamber $\left(D_{2} \simeq 2 \mathrm{~cm}\right)$ is much larger than the sample width justifying that the calculation of $h$, made in the cylindrical symmetry, is the most appropriate one [33]. Then in order to calculate $h_{1 r}$ and $h_{2 r}$ we need in fact to solve three problems, 1-find the right geometry for the system (fiber and chamber) allowing the use of the calculations of $h_{1 c}$ and $h_{2 c}$ in the symmetric cylindrical geometry (the subscript $c$ standing for cylindrical), 2-calculating $h_{1 c}$ and $h_{2 c}$ for various pressures, 3 -find the correspondence between the two geometry (rectangular and cylindrical), meaning a relation between $h_{1 c, 2 c}$ and $h_{1 r, 2 r}$. 


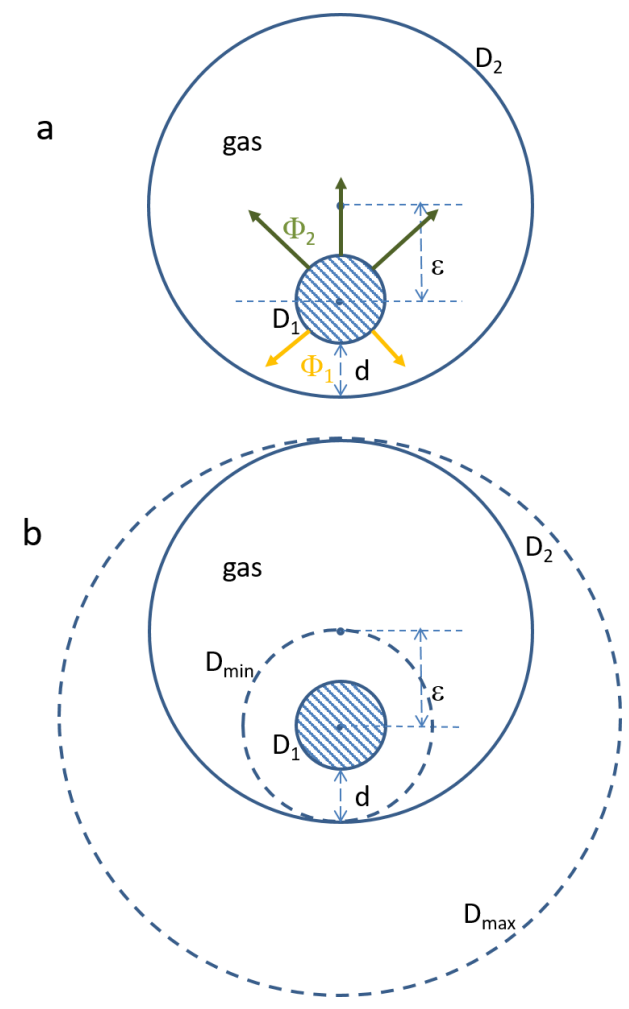

Fig. 6. Approximate model for the convection heat transport. a) $D_{1}$ is the diameter of the fiber (inner cylinder), $D_{2}$ is the diameter of the experimental chamber (outer cylinder), $\varepsilon$ is the separation between the center of the fiber and the center of the experiment chamber, $d$ is the distance from the bottom of the fiber to the bottom of the experimental chamber, $\Phi_{1}$ in orange and $\Phi_{2}$ in green are the heat flux respectively to the bottom or to the top of the chamber. b) schematic showing the assumption of the double cylindrical geometry where the heat flux to the top of the chamber $\Phi_{2}$ is calculated using the cylinder $D_{\max }$ and the heat flux to the bottom of the chamber $\Phi_{1}$ is calculated using the cylinder $D_{\min }$.

\subsection{1 $h_{1 c}$ and $h_{2 c}$ in a cylindrical geometry}

In a purely conductive regime, where $k_{\text {gas }}$ is the thermal conductivity of a gas filling the outer cylinder, the rate of total heat flow between the two cylinders of length $L$ and diameter $D_{1}$ and $D_{2}$, as depicted in Fig. 6 a, can be exactly calculated without any assumption on the geometry using the following equation [34]:

$$
\Phi=\pi D_{1} L \frac{k_{g a s}}{D_{1}} \frac{2\left(T_{2}-T_{1}\right)}{\cosh ^{-1}\left[\left(D_{1}^{2}+D_{2}^{2}-4 \varepsilon^{2}\right) / 2 D_{1} D_{2}\right]}=h_{c} S_{1} \Delta T
$$

where $\varepsilon$ is the vertical eccentricity of the inner cylinder and $S_{1}=\pi D_{1} L / 2$, the surface of the fiber.

A reasonable approximation can be made where the flux $\Phi$ is expressed as a sum of two heat fluxes. In this approximation, the heat flowing from the fiber to the surrounding chamber is divided in two parts. One part will be the upper heat flux, $\Phi_{2}$, that is exchanged between half of the surface $S_{1}$ with the top part of the chamber (approximated as a cylindrical chamber of diameter $D_{\max }=D_{2}+2 \varepsilon$ ), and the second part, the lower heat flux $\Phi_{1}$, that is exchanged between half of the surface $S_{1}$ with the bottom part of the chamber (approximated as a cylindrical chamber of diameter $D_{\min }=D_{1}+2 d$ ). The schematic describing this approximation is shown in Fig. 6 b. In each situation, the heat flux $\Phi_{1,2}$ will be associated with an exchange coefficient $h_{1 c, 2 c}$ following :

$$
\Phi_{1,2}=h_{1,2} \frac{S_{1}}{2} \Delta T
$$

knowing that the total heat flux is expressed through: $\Phi=\Phi_{1}+\Phi_{2}$. We can deduced from Eqn. 14 a mean value for $h$ :

$$
h_{c} \simeq \frac{h_{1 c}+h_{2 c}}{2}
$$


We can now compare the final value for $h$ that can be calculated using Eqn. 15 to the one calculated using the approximation made to obtain Eqn. $15 ; h_{1 c}$ and $h_{2 c}$ being calculated using Eqn. 13 replacing $D_{2}$ by $D_{\min }$ for $h_{1 c}$ and by $D_{\max }$ for $h_{2 c}$ along with $\varepsilon=0$ to be in accordance with our approximations.

Considering the typical sample prepared for this experiment, $D_{1}=9 \mu \mathrm{m}, d=71 \mu \mathrm{m}, D_{2}=2 \mathrm{~cm}, \varepsilon=1 \mathrm{~cm}$, $k_{\text {gas }}=2.4 \mathrm{~W} \cdot \mathrm{cm}^{-1} \cdot \mathrm{K}^{-1}$. The coefficient $h_{c}$ obtained from Eqn. 15 are then $\left(h_{1 c}+h_{2 c}\right) / 2=1292 \mathrm{~W} \cdot \mathrm{m}^{-2} \cdot \mathrm{K}^{-1}$ a very close value to the $h_{c}$ coefficient calculated from Eqn. 13: $h_{c}=1367 \mathrm{~W} \cdot \mathrm{m}^{-2} \cdot \mathrm{K}^{-1}$. Within a reasonable accuracy this calculation shows that our assumption of a double cylindrical geometry for the calculation of $h$ coefficients is good enough, and justifies its extension to the convection regime.

\subsubsection{The $h$ coefficients in the convective regime}

To estimate the convection coefficient, one needs also to discuss the different regimes of heat transfer through the gas since it will affect the limit used for the calculation. In these experiments, since pressure below the atmosphere is used, the heat transfer from the fiber to the gas will be done in the limit of rarefied gas; indeed, the case of atmospheric pressure is not the most interesting one for this study. By rarefied gas, we mean that the molecular mean free path $\lambda^{3}$ is not small as compared to the characteristic dimension $D$; here $D$ can be, for instance, the diameter of the filament $D_{1}=9 \mu \mathrm{m}$. Since the studies of Maxwell on this problem [35], numerous analytical solutions have been given, all of them depending on the Knudsen number $K n=\frac{\lambda}{D_{1}}$ [36]. Four different regimes of heat transfer can be distinguished: the free molecule regime $(K n \geq 10)$, the transition regime $(10 \geq K n \geq 0.1)$, the slip flow regime $(0.1 \geq K n \geq 0.01)$ and the continuum or hydrodynamic regime $(K n \leq 0.01)[33]$.

\subsection{3 $h_{1 r}$ and $h_{2 r}$ calculations in the rectangular geometry}

In this part, we will calculate $h_{1 r}$ and $h_{2 r}$ convection coefficients calculated in rectangular conditions as used in the final equations describing the temperature oscillation Eqn. 6, Eqn. 7 and Eqn. 8 as a function of $h_{1 c}$ and $h_{2 c}$ convection coefficients calculated in cylindrical conditions. In the calculation of the $\left(V_{3 \omega}\right)_{r m s}$ we used a model where the area of the normal section of the sample was $2 a b$ (see Fig. 5), but the real sample is a cylinder with diameter $D_{1}$, both of them have the same length $L$. If we take $2 a=\pi D_{1} / 2$ and $b=D_{1} / 2$, we have the same density of power per $\mathrm{m}^{3}$ in cylindrical or rectangular condition, which is necessary in vacuum conditions. But when the surface of the sample exchanges power with gas, our approximation is working, as we explained, if $a b=\pi D_{1}^{2} / 8$ along with $\frac{\pi D_{1} L h_{2 c}}{2}=2 a L h_{2 c}=(2 a+2 b) L h_{2 r}$, then a simple geometrical correspondence gives the relation between $h_{1 r}, h_{2 r}$ and $h_{1 c}, h_{2 c}$ :

$$
\begin{gathered}
h_{1 r}=h_{1 c} \\
h_{2 r}=\frac{h_{2 c}}{1+\frac{2}{\pi}}
\end{gathered}
$$

Now, in the following, $h_{1 c}$ and $h_{2 c}$ will be calculated for different ranges of pressure. For a diatomic gas in the free molecule regime and in the transition regime (low pressure) the convection coefficient is given by [33]:

$$
h_{1,2}^{L P}=\frac{h_{F M}}{1+\frac{4 \times 45 \times 0.5 \alpha_{r} D_{1}}{15 \times 38 \lambda} \ln \left(\frac{D_{\min , \max }}{D_{1}}\right)}
$$

$D_{\min , \max }$ being defined as in Fig. 6. In Eqn. $18, h_{F M}$ is the convection coefficient in the free molecule regime given by:

$$
h_{F M}=\frac{\alpha_{r}(\gamma+1)}{2(\gamma-1)} \sqrt{\frac{R_{0}}{2 \pi M T_{2}}} p
$$

\footnotetext{
${ }^{3}$ The mean free path at a pressure $P$ (as compared to the atmospheric pressure $P_{a}$ ) is given by the following equation $\lambda=5.88 \times$ $10^{-3} P_{a} / P \sqrt{\frac{T}{T_{0}}}$ where $T_{0}$ is the room temperature.
} 


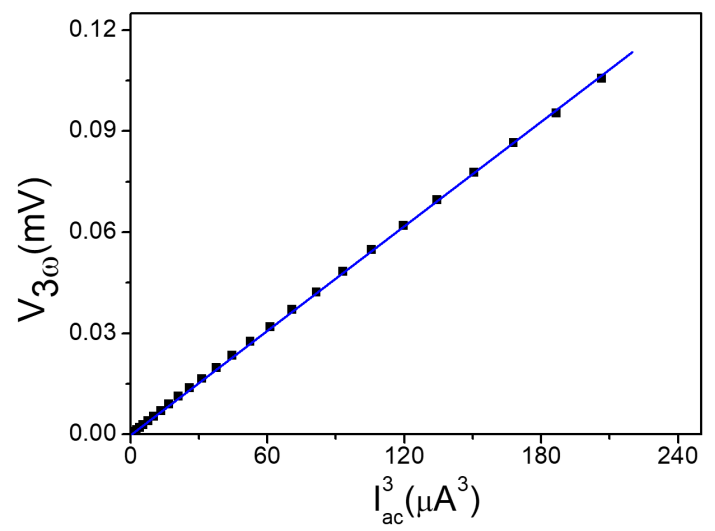

Fig. 7. $V_{3 \omega}$ versus RMS heating current at the frequency of $1.3 \mathrm{~Hz}$. The linearity of the voltage signal measured by the lock-in amplifier with the cube of the RMS current is showing that no over-heating is present during the measurement.

where $\alpha_{r}=\frac{\alpha}{1+(1-\alpha) \frac{D_{1}}{D_{2}}}$ with $\alpha$ is the accommodation coefficient closed to 0.93 (see Appendix A), $\gamma$ is the ratio of the specific heats at constant pressure and constant volume (for $\mathrm{N}_{2} \gamma=1.4$ ), $M$ is the molecular mass of $\mathrm{N}_{2}$ in $\mathrm{kg}$ per mole, $T_{2}$ is the temperature of the measurement $\left(T_{2}=298 \mathrm{~K}\right), p$ is the pressure of the gas in Pascal and $R$ is the molar gas constant $\left(R=8.314 \mathrm{~J} \cdot \mathrm{mol}^{-1} \cdot \mathrm{K}^{-1}\right)$.

In the slip flow regime, with $p \geq 3 \mathrm{hPa}$ (corresponding to a mean free path $\lambda=20 \mu \mathrm{m}$ ), we used a calculation proposed by Hadj-Nacer et al. [37]:

$$
h_{1,2}^{H P}=\frac{15 \lambda p}{4 D_{1}} \sqrt{\frac{2 T_{2} R_{0}}{M}} \frac{2\left(T_{1}^{1.5}-T_{2}^{1.5}\right)}{3 T_{2}^{1.5}\left(T_{1}-T_{2}\right)\left[\ln \left(\frac{D_{\min , \max }}{D_{1}}\right)+A \frac{2 \lambda}{D_{1}}\left(\frac{T_{1}}{T_{2}}+\frac{D_{1}}{D_{\min , \max }}\right)\right]}
$$

with $A=\left(\frac{2-\alpha}{\alpha}+0.17\right) \frac{\sqrt{\pi}}{P_{r}} \frac{\gamma}{\gamma+1}$ where $P_{r}$ is Prandtl number, for $\mathrm{N}_{2}$ at $T_{2}=298 \mathrm{~K} P_{r}=0.71$. For each range of pressure, $h_{L P}^{1,2}$ and $h_{H P}^{1,2}$ have to be calculated for different surfaces of the samples.

As a conclusion for this part, depending of the regime of pressure, $h_{1 c}$ and $h_{2 c}$ will be equaled to either $h_{1,2}^{L P}$ in the low pressure regime or $h_{1,2}^{H P}$ in the high pressure regime as described in Eqn. 18 and Eqn. 20.

\section{Results}

4.1 Thermal conductivity and specific heat of glass fiber

The first test we have to do in the measurement of thermal conductivity and specific heat of glass fiber is to measure the dependency of the $3 \omega$ signal on the amplitude of the applied RMS current. Fig. 7 represents the $3 \omega$ voltage signal as a function of $I_{a c}^{3}$. The data shows a linear behavior meaning that in this measurement range the fiber is not over-heated. This linear dependency with the cube of the current is the confirmation of the proper functioning of the method and device. Then to extract the thermal properties $\left(k\right.$ and $\left.c_{p}\right)$, the $V_{3 \omega}$ signal is measured as a function of the frequency of the heating current as shown in Fig. 8. Red circles represent the experiment data, black line is the adjustment of the data by using Eqn. 12 with fitting parameters $k$ and $c_{p}$. One can noticed that the device has the expected response to the change of frequency as predicted by $3 \omega$ technique modelling through Eqn. 12 giving high confidence in the thermal conductivity and specific heat extracted from the experiment.

The measurements have been performed on many different fibers having various sizes. For each one, we fit the experimental data to get the thermal conductivity and specific heat of the glass fiber as the fitting parameters using Eqn. 12. By doing a statistical analysis on all the devices (more than twenty samples were measured), we have obtained an average value of the thermal conductivity of glass fiber about $1.08 \pm 0.12 \mathrm{~W} \cdot \mathrm{m}^{-1} \cdot \mathrm{K}^{-1}$ and specific heat about $0.78 \pm 0.17 \mathrm{~J} \cdot \mathrm{g}^{-1} \cdot \mathrm{K}^{-1}$, which are in very good agreement with the values found in literature [38-41]. It can be noticed that the thermal conductivity is obtained with little scattering, though the specific heat shows a larger dispersion. This is coming from the fact that in the $3 \omega$ technique, thermal conductivity is measured with lesser experimental error than the specific heat since it is estimated by fitting the high frequency part of the $V_{3 \omega}$ measurement $[42,43]$. 


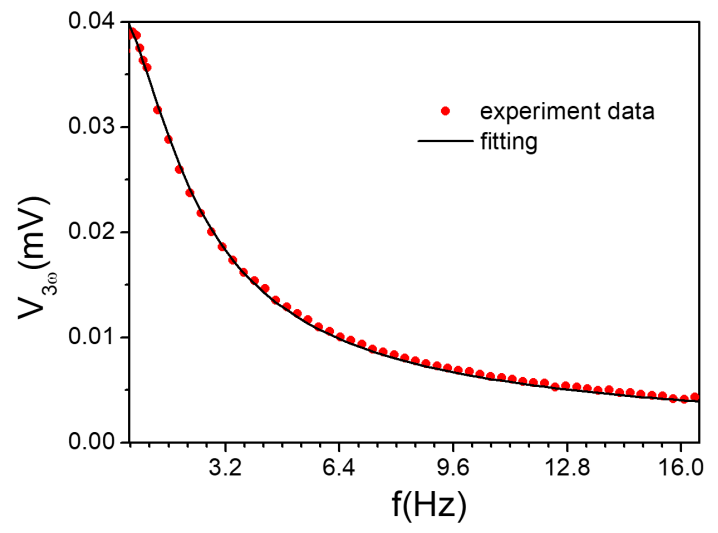

Fig. 8. $V_{3 \omega}$ voltage as a function of the heating current frequency for bias current of $I=3.94 \mu \mathrm{A}$. The red circles are the experimental data and the black line is the fitting curve using Eqn. 12 that will give the values of thermal conductivity and specific heat; in the case of this sample, we find $k=1.05 \mathrm{~W} \cdot \mathrm{m}^{-1} \cdot \mathrm{K}^{-1}$ and $c_{p} 0.80 \mathrm{~J} . \mathrm{g}^{-1} \cdot \mathrm{K}^{-1}$.

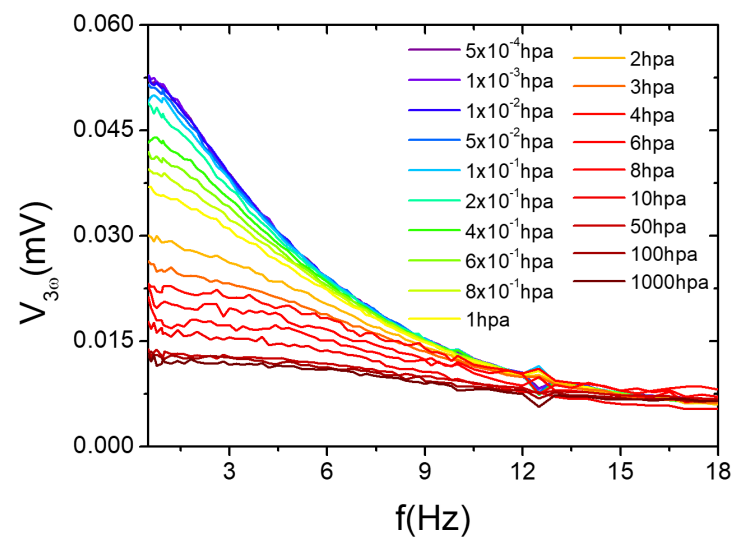

Fig. 9. $\quad V_{3 \omega}$ signal versus frequency at different gas pressures. The $V_{3 \omega}$ is first collected at high vacuum, then the measurement chamber is filled gradually with $\mathrm{N}_{2}$. At each controlled pressure, $V_{3 \omega}$ is measured as function of frequency.

Figure 9 shows a set of $V_{3 \omega}$ voltages as a function of frequency measured for different pressures of $\mathrm{N}_{2}$ from high vacuum $\left(10^{-4} \mathrm{hPa}\right.$ to ambient pressure $\left.1000 \mathrm{hPa}\right)$. These data clearly show a dependence of the $V_{3 \omega}$ signals to the pressure of the gas; the $V_{3 \omega}$ voltages decreasing as the pressure increases due to a decrease of the temperature oscillation. This is expected since by adding gas an extra thermalization path is provided.

To perform the $V_{3 \omega}$ data treatment using Eqn. 12, we will set the value of $h_{1 r}$ to the one that is calculated from the model described in the prior section using Eqn. 16, Eqn. 18 and Eqn. 20, keeping $h_{2 r}$ as the only free parameter. For obtaining the convection coefficient $h_{2 r}$ at various pressure, the procedure described below has been used. First, under vacuum condition $\left(h_{1 c}=h_{2 c}=0\right)$, the fitting of $\left(V_{3 \omega}\right)_{r m s}$ will give the intrinsic thermal properties of the glass fiber $k$ and $c_{p}$, and second for experiments done under gas pressure, the $\left(V_{3 \omega}\right)_{r m s}$ is fitted using Eqn. 12 with the convection coefficient $h_{2 r}$ as free parameter. The comparison of the experimental value of $h_{2 r}$ with the model required to consider two situations. In the low pressure case, when $p \leq 3 \mathrm{hPa}$, the experimental value $h_{2 r}$ is compared to the calculated value $h_{2}^{L P}$ as obtained from Eqn. 17 and Eqn. 18. For the high pressure limit, when $p \geq 3 \mathrm{hPa}$ the experimental value $h_{2 r}$ is compared to the calculated value $h_{2}^{H P}$ given by Eqn. 17 and Eqn. 20.

These results are presented in Fig. 10. There is a very good agreement on all the measurement ranges of pressure between the value of $h_{2}$ obtained by fitting the Eqn. 12 to our experimental data, and $h_{2}$ calculated with Eqn. 18 or Eqn. 20. As a conclusion, for a wire of about $10 \mu \mathrm{m}$, the variation of the convection coefficients with pressure can be well described by classical analysis of convection processes. At atmospheric pressure, a value of $300 \mathrm{~W} \cdot \mathrm{m}^{-2} \cdot \mathrm{K}^{-1}$ is found for the convective coefficient much bigger than for macroscale surface where the $h$ coefficient is equal to $10 \mathrm{~W} \cdot \mathrm{m}^{-2} \cdot \mathrm{K}^{-1}[4,44]$. This value can be compared to several existing values in the literature of microfabricated structures from $35 \mathrm{~W} \cdot \mathrm{m}^{-2} \cdot \mathrm{K}^{-1}$ measured on membranes by Jain et al. [45] and the one found 


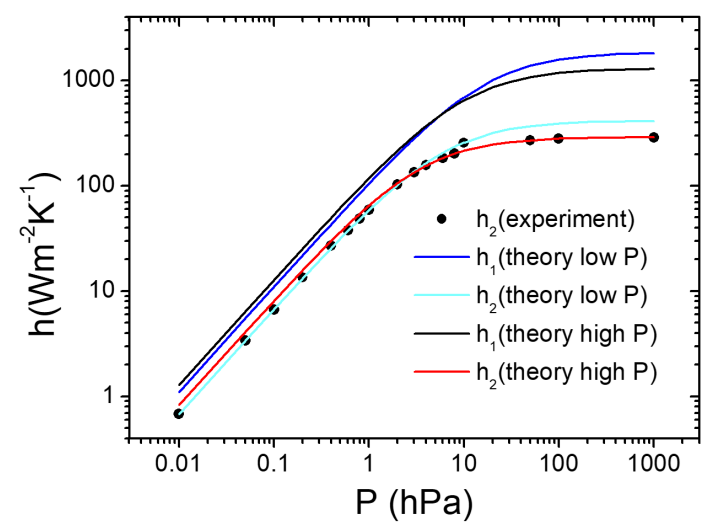

Fig. 10. Convection coefficients obtained by fitting our experimental data in comparison to the calculated values obtained from model detailed in the present work. The black circles represent the convection coefficient $h_{2 r}$ obtained from the fit of the experimental data; the calculated coefficients $h_{1}$ and $h_{2}$ from the model are also presented for comparison: the black line is the convection coefficient $h_{1}$ in the high pressure limit, the dark blue line corresponds to $h_{1}$ in the low pressure limit; the red line is the convection coefficient $h_{2}$ obtained for high pressure, and the light blue line for low pressure.

by Gao et al. where a coefficient of $600 \mathrm{~W} \cdot \mathrm{m}^{-2} \cdot \mathrm{K}^{-1}$ has been measured on suspended platinum wire [25]; values even much higher have been found in the case of carbon nanotubes [46]. The difference could originate from the fact that the different micro or nano-structures do not have the same surface roughness and dimensions, but also because Gao and coworkers, for instance, have used an experiment involving much higher $\Delta T$ along with different data treatment techniques. As a conclusion, this experiment is of interest not only to evaluate $h$ factor in the case of MEMs and suspended probes for scanning microscopy, but also to show that a suspended glass fiber may serve as a possible sensitive device for gas sensing for pressure $p$ below $10 \mathrm{hPa}$.

4.2 Thermal contact resistance between two glass fibers

The last part of this work is dedicated to the estimation of the contact thermal resistance that may exist between two glass fibers. This thermal resistance is playing a significant role in all the applications for thermal isolation involving glass fibers, especially in rock-wool materials. The following is then detailing the experiment and its modelling along with the experimental results.

\subsubsection{Thermal contact resistance model}

In order to get access to the thermal resistance when two glass fibers are put in contact, few suspended glass fibers are installed in parallel. Then, we select two suspended fibers one next to the other, and on these two fibers, we place another fiber at the middle of the two fibers as shown in Fig. 11. This configuration of the three fibers forms a H-like structure, referred to as H-type probe in the following [47]. In this measurement, one suspended fiber will be used as heating wire $\left(L_{1}\right)$, and the other one acts as sensing wire $\left(L_{2}\right)$. By measuring the temperature of the heating wire and sensing wire at the thermal equilibrium state, we can determine the thermal contact resistance between the top fiber and the two fibers underneath as depicted in Fig. 11; this means that we will actually measure the thermal contact between a glass fiber coated with $\mathrm{NbN}$ and a bare glass fiber.

In this development, a DC current is first applied to the heating wire $\left(L_{1}\right)$. Due to Joule heating, the fiber $L_{1}$ will be heated to an equilibrium temperature with $T_{1 \max }=T_{1}$ at the center of the fiber $L_{1}$. Then due to the heat transfer through the crossing fiber, the fiber $L_{2}$ is slightly heated to an equilibrium state with the maximum temperature at the center $T_{2 \max }=T_{2}$. By measuring the temperature $T_{1}$ and $T_{2}$, the thermal contact resistance can be extracted.

The thermal model of the contact resistance is detailed below. Based on the thermal resistance circuit presented in Fig. 12, the heat flux $\dot{Q}_{c}$ from fiber $L_{1}$ to fiber $L_{2}$ through the crossing fiber $L_{3}$ is given by:

$$
\dot{Q}_{c}=\frac{T_{1}-T_{2}}{2 R_{c}+R_{3}}
$$

where $R_{c}$ and $R_{3}$ are the thermal contact resistances between glass fibers and thermal resistance of fiber $L_{3}$ 


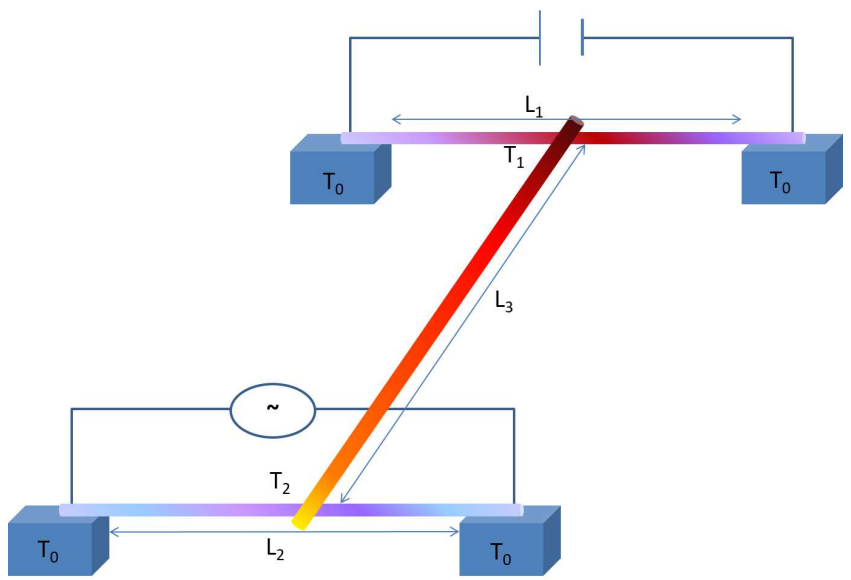

Fig. 11. Scheme of the $\mathrm{H}$-type experiment: $L_{3}$ is the crossing fiber (of $1.05 \mathrm{~mm}$ length) connecting thermally the two other fibers $L_{1}=$ $0.39 \mathrm{~mm}$ and $L_{2}=0.5 \mathrm{~mm} ; L_{1}$ is heated by a DC current, a voltmeter is used to measure the voltage on the heating fiber and get its temperature $T_{1}$; the sensing fiber $L_{2}$ is excited by an AC current, and a lock-in amplifier is used to measure the voltage on the sensing fiber and gets its temperature $T_{2}$. The temperature gradient along the fibers will determine the thermal resistance of the contact between the fibers.

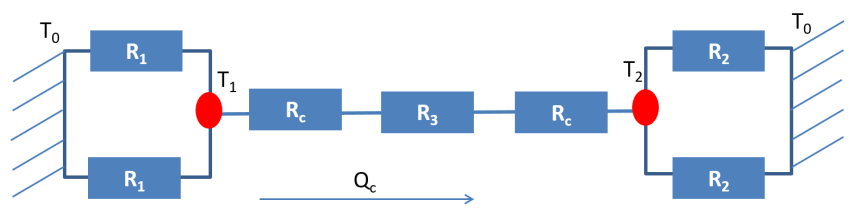

Fig. 12. Thermal resistance circuit representing the $\mathrm{H}$-type experiment. $T_{0}$ is the temperature of the thermal bath, $T_{1}$ is the temperature at the contact between fiber $L_{1}$ and fiber $L_{3}, T_{2}$ the temperature at the contact between fiber $L_{2}$ and fiber $L_{3}, R_{c}$ is the thermal contact resistance between two glass fibers which is supposed to be the same for different fiber pairs, $R_{1}$ and $R_{2}$ are halves of the thermal resistance of the fibers $L_{1}$ and $L_{2}$, and $R_{3}$ is the thermal resistance of the fiber $L_{3}$.

respectively. Then the heat flux through the sensing fiber $L_{2}$ to thermal bath is given by:

$$
\dot{Q}_{2}=\frac{2\left(\mathrm{~T}_{2}-\mathrm{T}_{0}\right)}{\mathrm{R}_{2}}
$$

where $R_{2}$ is a half of the thermal resistance of the fiber $L_{2}$. Since the heat flux through $L_{3}$ is equal to the heat flux through $L_{2}$ then:

$$
\frac{T_{1}-T_{2}}{2 R_{c}+R_{3}}=\frac{2\left(T_{2}-T_{0}\right)}{R_{2}} \Rightarrow R_{c}=\frac{R_{2}\left(T_{1}-T_{2}\right)}{4\left(T_{2}-T_{0}\right)}-\frac{R_{3}}{2}
$$

Substituting $R_{3}=L_{3} /\left(k_{3} A_{3}\right)$ in to the above equation, the thermal contact resistance is found to be:

$$
R_{c}=\frac{L_{2}\left(T_{1}-T_{2}\right)}{8 k_{2} A_{2}\left(T_{2}-T_{0}\right)}-\frac{L_{3}}{2 k_{3} A_{3}}
$$

where $k_{3}, A_{3}$ and $k_{2}, A_{2}$ are the thermal conductivities and cross-section of the crossing-fiber $L_{3}$ and the sensing fiber $L_{2}$ respectively. Assuming that the fibers have the same thermal conductivity and diameter $\left(k_{2}=k_{3}\right.$ and $\left.A_{2}=A_{3}\right)$, the thermal contact resistance is written in a simpler form:

$$
R_{c}=\frac{1}{2 k_{3} A_{3}}\left(\frac{L_{2}\left(T_{1}-T_{2}\right)}{4\left(T_{2}-T_{0}\right)}-L_{3}\right)
$$




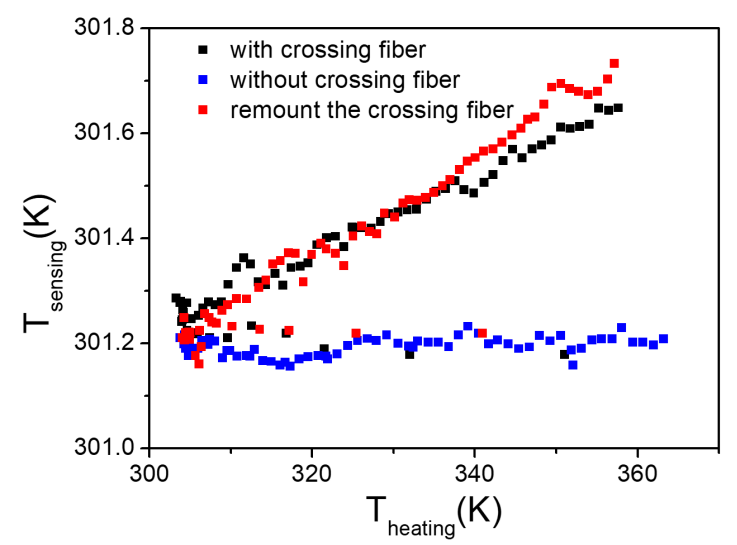

Fig. 13. Temperature on the sensing side versus temperature on the heating side when a fiber is deposited across the two other fibers. The blue squares represent data after removing the crossing-fiber to serve as a reference, as expected, no heating can be detected. The red squares represent data after re-mounting the crossing-fiber to show the reproducibility of the experiment.

Since $k_{3}, A_{3}, L_{2}, L_{3}$ and $T_{0}$ are known parameters, when $T_{1}$ and $T_{2}$ are measured, the thermal contact resistance can be calculated by using Eqn. 25 .

\subsubsection{Thermal contact resistance between glass fibers}

In the experimental set up to estimate the thermal contact resistance, we sweep the heating current $I_{D C}$ from $1 \mu \mathrm{A}$ to $70 \mu \mathrm{A}$ with step of $1 \mu \mathrm{A}$ and collect the temperature on both heating and sensing sides. The data is shown in Fig. 13. From this data, we can clearly see heating effect on the sensing fiber with the appearance of the crossing-fiber (black squares in Fig. 13). This heating effect is confirmed to be due to the heat transfer through the crossing-fiber, since the sensing fiber is not heated when the fiber bridging the two others is not present (blue squares in Fig. 13). The reproducibility is confirmed by mounting again the crossing-fiber (red squares in Fig. 13) and obtaining the same results.

From the data presented in Fig. 13, we applied the Eqn. 25, with the known dimension of the fiber as well as the thermal conductivity of glass fiber $\left(k=1.1 \mathrm{~W} \cdot \mathrm{m}^{-1} \cdot \mathrm{K}^{-1}\right)$ to obtain the thermal contact resistance

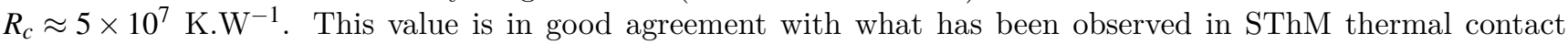
measurements $[48,49]$. While the crossing cylindrical fibers between which the contact is made in our experiment have a diameter of $9 \mu \mathrm{m}$, their surface exhibits sub-nanometer roughness over micrometer distances due to the drawing process by which they are produced [50]. It is thus likely that they are in contact only through nanometersized channels in vacuum. Note that the thermal contact resistance measurement that we perform does not discriminate between the conductive and the near-field radiative channel. However, it is expected that the latter will contribute very little since experimental measurements of the radiative thermal conductance as a function of the distance between two objects exhibits a dramatic increase when solid-solid contact is established [51,52].

\section{Conclusion}

We have developed an experimental method for the measurements of thermal conductivity and specific heat of a single suspended glass fiber, thermal contact resistance between two glass fibers and convection through the exchange gas at different gas pressures. The measurements based on the $3 \omega$ technique have shown that thermal conductivity and heat capacity of glass fiber are the same as that of regular glass $\left(k=1.1 \mathrm{~W} \cdot \mathrm{m}^{-1} \cdot \mathrm{K}^{-1}\right.$, $\left.c_{p}=0.79 \mathrm{~J}_{\mathrm{g}} \mathrm{g}^{-1} \cdot \mathrm{K}^{-1}\right)$. The convection heat transfer was analyzed by changing the pressure of the exchange gas in the measurement chamber. With the same sample, it was possible to perform different measurements and get the contribution in thermal transport from different heat transfer mechanisms in single glass fiber. The $3 \omega$ experiment allowed to extract the convection coefficient from the fiber to the rest of the experimental chamber at different pressures in good agreement with the various models of heat transfer through a gas. Finally, by using an H-type technique, the thermal contact resistance has been measured with a value in the order of $5 \times 10^{7} \mathrm{~K} . \mathrm{W}^{-1}$. These observations will contribute to estimate which heat transfer mechanisms is playing the key role in insulation material (conduction, convection or contact resistance). 
Acknowledgements

The authors thank the technical support and discussions provided by the Nanofab, Pôle Capteur, Pôle électronique, Pôle cryogenic facilities in Institut Néel. The authors acknowledge support the Laboratoire d'excellence LANEF in Grenoble (No. ANR-10-LABX-51-01), and from Saint Gobain Research Paris for providing the glass fibers. This work also received financial support from LABEX WIFI (Laboratory of Excellence within the French Program Investments for the Future) under references ANR-10-LABX-24 and ANR-10- IDEX-0001-02 PSL*, and Agence Nationale de la Recherche (ANR), Project CarISOVERRE under reference ANR-16-CE09-0012.

Appendix A: Coefficient of thermal accommodation

In order to calculate the heat transfer from the heater, it is necessary to know the flux of energy and momentum carried by the molecules of gas impinging on the surface and then reflected from it. A coefficient of thermal accommodation is then defined as $[33,36]$ :

$$
\alpha=\frac{T_{r}-T_{i}}{T_{S}-T_{i}}
$$

with $T_{r}$ and $T_{i}$ are the temperatures of the reflected and incident gas streams, respectively; $T_{s}$ is the temperature the molecular stream leaving the surface would have, if this stream were to carry the same mean energy as a stream issued from a gas in equilibrium at $T_{s}$. As an example, from experimental data obtained for a fiber of $\mathrm{W}$ in nitrogen gas: $0.6 \leq \alpha \leq 0.95$ at room temperature [33].

\section{Nomenclature}

$a$ Half width of the thermometric $\mathrm{NbN}$ strip.

$b$ Thickness of the $\mathrm{SiO}_{2}$ fiber in the rectangular approximation.

$c_{p}$ Specific heat.

$d$ Distance from the bottom of the glass fiber to the Si surface.

$e$ Thickness of the NbN strip.

$f$ Frequency of the electrical current.

$h$ Convection coefficients, generic name.

$h_{1}$ Convection coefficient of the bottom of the fiber.

$h_{2}$ Convection coefficient of the top and sides of the fiber.

$h_{1 c}$ Convection coefficient $h_{1}$ in cylindrical condition.

$h_{2 c}$ Convection coefficient $h_{2}$ in cylindrical condition.

$h_{1 r}$ Convection coefficient $h_{1}$ in rectangular condition.

$h_{2 r}$ Convection coefficient $h_{2}$ in rectangular condition.

$h_{1,2}^{L P} \quad$ Convection coefficient $h_{1,2}$ in low pressure condition.

$h_{1,2}^{H P}$ Convection coefficient $h_{1,2}$ in high pressure condition.

$k$ Thermal conductivity.

$k_{2}$ Thermal conductivity of fiber $L_{2}$.

$k_{3}$ Thermal conductivity of fiber $L_{3}$.

$p$ Pressure in the measurement chamber.

$x, y, z$ and $t$ Space and time coordinates.

$A_{2} \quad$ Cross section of fiber $L_{2}$.

$A_{3}$ Cross section of fiber $L_{3}$.

$D_{\text {diff }}$ The diffusivity of the fiber materials.

$D_{1}$ Diameter of the glass fiber.

$\mathrm{D}_{2}$ Diameter of the measurement chamber.

I Electrical current.

$I_{0} \quad$ Amlitude of the electrical current.

$I_{r m s}$ Root mean square of the electrical current.

$K n$ Knudsen number.

$L$ Length of the glass fiber.

$L_{1} \quad$ Length of the glass fiber 1 .

$L_{2} \quad$ Length of the glass fiber 2 . 
$L_{3} \quad$ Length of the glass fiber 3.

$M$ The molecular mass.

$P$ Electrical power.

$P_{a}$ Pressure in the measurement chamber.

$P_{a c}$ The oscillating part of the heating power.

$P_{d c} \quad$ The constant part of the heating power.

$P_{0} \quad$ Amplitude of the electrical power.

$\dot{Q}_{2} \quad$ Heat flux through the fiber $L_{2}$.

$\dot{Q}_{c} \quad$ Heat flux through the fiber $L_{3}$.

$R$ The molar gas constant.

$R_{0} \quad$ Resistance of the transducer at $T=T_{0}$.

$R_{1}$ Half of the thermal resistance of the fiber $L_{1}$.

$R_{2}$ Half of the thermal resistance of the fiber $L_{2}$.

$R_{3}$ Thermal resistance of fiber $L_{3}$.

$R_{c}$ Thermal contact resistance.

$R_{t h}$ Resistance of the transducer as function of time.

$S_{1} \quad$ Surface area of the fiber.

$T$ Temperature.

$T_{0}$ Temperature of the thermal bath.

$T_{1}$ Temperature at the contact between fiber $L_{1}$ and fiber $L_{3}$.

$T_{1 \max }$ Maximum of the temperature distribution on the fiber $L_{1}$ due to Joule heating.

$T_{2 \max }$ Maximum of the temperature distribution on the fiber $L_{2}$.

$T_{2} \quad$ Temperature at the contact between fiber $L_{2}$ and fiber $L_{3}$.

$T_{a c}$ The oscillating part of temperature gradient.

$T_{d c}$ The constant part of the temperature gradient.

$T_{\text {heating }}$ the temperature on the heating fiber $L_{1}$.

$T_{i}$ Temperatures of the incident gas stream.

$T_{i} m$ The imaginary part of the temperature.

$T_{r}$ temperatures of the reflected gas stream.

$T_{\text {real }}$ The real part of the temperature.

$T_{s}$ The temperature of the molecular stream leaving the surface.

$T_{\text {sensing }}$ The temperature on the sensing fiber $L_{2}$.

$T_{\text {stat }}$ Stationary part of the temperature.

$V_{3 \omega} \quad 3 \omega$ voltage signal.

$\left(V_{3 \omega}\right)_{r m s}$ Root mean square of the $3 \omega$ voltage signal.

$U$ Transient part of the temperature gradient.

$U_{h}$ Periodic part of the temperature oscillation.

$\bar{U}_{h} \quad$ The complex mean temperature.

$U_{r}$ The mean temperature in the real space.

$\alpha_{T C R}$ Temperature coefficient of the resistance.

$\alpha$ Coefficient of thermal accomodation.

$\gamma$ The ratio of the specific heats at constant pressure and constant volume.

$\lambda$ Molecular mean free path.

$\varepsilon$ The separation between the center of the fiber and the center of the experiment chamber/vertical eccentricity

$\omega$ Angular frequency of the heating current.

$\phi \quad$ Phase lag of the $3 \omega$ voltage signal.

$\rho$ Mass density of the fiber.

$\Phi$ Total heat flow between the fiber and the chamber.

$\Phi_{1} \quad$ Total heat flow between two concentric cylinders with diameters $D_{1}$ and $D_{\min }=D_{1}+2 \varepsilon$.

$\Phi_{2}$ Total heat flow between two concentric cylinders of diameters $D_{1}$ and $D_{\max }=D_{1}+2 d$.

References

[1] Allouhi, A., Fouih, Y. E., Kousksou, T., Jamil, A., b, Y. Z., and Mourad, Y., 2015. "Energy consumption and efficiency in buildings: current status and future trends". J. Cleaner Prod., 109, pp. 118-130.

[2] Tanica, M., Stankovica, D., Nikolica, V., Nikolica, M., Kostica, D., Milojkovica, A., Spasica, S., and Vatin, N., 2015. "Reducing energy consumption by optimizing thermal losses and measures of energy recovery in preschools". Procedia Engineering, 117, pp. 919-932. 
[3] Jelle, B., 2011. "Traditional, state-of-the-art and future thermal building insulation materials and solutions - properties, requirements and possibilities". Energy Build, 43, pp. 2549-2563.

[4] Langlais, C., and Klarsfeld, S., 2004. "Isolation thermique à température ambiante. propriétés." Techniques de l'Ingénieur, 9, p. 960.

[5] Cahill, D. G., 1990. "Thermal conductivity measurement from 30 to $750 \mathrm{k}$ : the $3 \omega$ method". Rev. Sci. lnstrum., 61, p. 802.

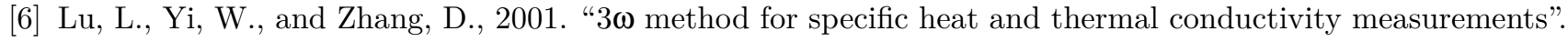
Rev. Sci. lnstrum., 72, pp. 2996-3003.

[7] Choi, T. Y., Poulikakos, D., Tharian, J., and Sennhauser, U., 2006. "Measurement of the thermal conductivity of individual carbon nanotubes by the four-point 3 $\omega$ method". Nano Lett., 6, pp. 1589-1593.

[8] Hou, J., Wanga, X., Vellelacheruvu, P., and Guo, J., 2006. "Thermal characterization of single-wall carbon nanotube bundles using the self-heating $3 \omega$ technique". J. Appl. Phys., 100, p. 124314.

[9] Bourgeois, O., Fournier, T., and Chaussy, J., 2007. "Measurement of the thermal conductance of silicon nanowires at low temperature". J. Appl. Phys., 101, p. 016104.

[10] Heron, J. S., Fournier, T., Mingo, N., and Bourgeois, O., 2009. "Mesoscopic size effects on the thermal conductance of silicon nanowire". Nano Lett., 9, pp. 1861-1965.

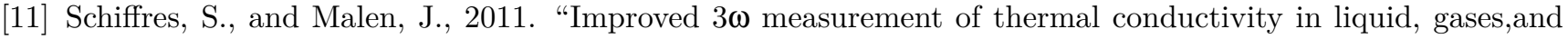
powders using a metal-coated optical fiber". Rev. Sci. lnstrum., 82, p. 064903.

[12] Sikora, A., Ftouni, H., Richard, J., Hébert, C., Eon, D., Omnes, F., and Bourgeois, O., 2012. "Highly sensitive thermal conductivity measurements of suspended membranes (sin and diamond) using a $3 \omega$-völklein method". Rev. Sci. Instrum., 83(5), p. 054902.

[13] Sikora, A., Ftouni, H., Richard, J., Hébert, C., Eon, D., Omnes, F., and Bourgeois, O., 2013. "Erratum: "highly sensitive thermal conductivity measurements of suspended membranes (sin and diamond) using a $3 \omega$ völklein method" [rev. sci. instrum. 83, 054902 (2012)]". Rev. Sci. Instrum., 84, p. 029901.

[14] Finefrock, S. W., Wang, Y., Ferguson, J. B., Ward, J. V., Fang, H., Pfluger, J. E., Dudis, D. S., Ruan, X., and $\mathrm{Wu}, \mathrm{Y} ., 2013$. "Measurement of thermal conductivity of pbte nanocrystal coated glass fibers by the $3 \omega$ method". Nano Lett., 13, pp. 5006-5012.

[15] Xing, C., Jensen, C., Munro, T., White, B., Ban, H., and Chirtoc, M., 2014. "Accurate thermal property measurement of fine fibers by the $3 \omega$ technique". Appl. Therm. Eng., 73, pp. 317-324.

[16] Xing, C., Jensen, C., Munro, T., White, B., Ban, H., and Chirtoc, M., 2014. "Thermal property characterization of fine fibers by the $3 \omega$ technique". Appl. Therm. Eng., 71, pp. 589-595.

[17] Munro, T., Xing, C., Ban, H., Copeland, C., Lewis, R., and Glorieux, C. "Thermal property measurement of thin fibers-a direct approach".

[18] Kommandur, S., Mahdavifar, A., Hesketh, P. J., and Yee, S., 2015. "A microbridge heater for low power gas sensing based on the $3 \omega$ technique". Sens. and Actuators A, 233, pp. 231-238.

[19] Hou, J., Wang, X., and Guo, J., 2006. "Thermal characterization of micro/nanoscale conductive and nonconductive wires based on optical heating and electrical thermal sensing.". J. Phys. D: Appl. Phys., 39, pp. 3362-3370.

[20] Guo, J., Wang, X., Geohegan, D. B., Eres, G., and Vincent, C., 2008. "Development of pulsed laser-assisted thermal relaxation technique for thermal characterization of microscale wires". J. Appl. Phys., 103, p. 113505.

[21] Guo, J., Wang, X., Geehegan, D. B., and Eres, G., 2008. "Thermal characterization of multi-wall carbon nanotube bundles based on pulsed laser-assisted thermal relaxation". Funct. Mater. Lett., 1, pp. 71-76.

[22] Guo, J., Wang, X., and Wang, T., 2007. "Thermal characterization of microscale conductive and nonconductive wires using transient electrothermal technique". J. Appl. Phys., 101, p. 063537.

[23] Park, B. K., Park, J., and Kim, D., 2010. "Note: Three-omega method to measure thermal properties of subnanoliter liquid samples". Rev. Sci. Instrum., 81, p. 066104.

[24] Lee, S. M., 2009. "Thermal conductivity measurement of fluids using the $3 \omega$ method". Rev. Sci. Instrum., 80, p. 024901.

[25] Gao, J., Xie, D., Xiong, Y., and Yue, Y., 2018. "Thermal characterization of microscale heat convection in rare-gas environment by a steady state "hot wire" method". Appl. Phys. Express, 11, p. 066601.

[26] Yang, J., Waltermire, S., Chen, Y., Zinn, A. A., Xu, T., and Li, D., 2010. "Contact thermal resistance between individual multiwall carbon nanotubes". Appl. Phys. Lett., 96, p. 023109.

[27] Guo, L., Wang, J., Lin, Z., Gacek, S., and Wang, X., 2009. "Anisotropic thermal transport in highly ordered tio2 nanotube arrays". J. Appl. Phys., 106, p. 123526.

[28] Wang, J., Song, B., Gu, M., and Zhang, X., 2010. "Temperature dependence of thermal resistance of a bare joint". Int. J. Heat Mass Trans., 53, pp. 5350-5354.

[29] Wang, J. L., Gu, M., Zhang, X., and Song, Y., 2009. "Thermal conductivity measurement of an individual fibre using a t type probe method". J. Phys. D: Appl. Phys., 42, p. 105502. 
[30] Wang, J., Gu, M., Zhang, X., and Wu, G., 2009. "Measurements of thermal effusivity of a fine wire and contact resistance of a junction using a t type probe". Rev. Sci. Instrum., 80, p. 076107.

[31] Bourgeois, O., André, E., Macovei, C., and Chaussy, J., 2006. "Liquid nitrogen to room-temperature thermometry using niobium nitride thin films". Rev. Sci. Instrum., 77, p. 126108.

[32] Carslaw, H., and Jaeger, J., 1959. Conduction of heat in solids. Oxford at Clarendon Press.

[33] Demirel, Y., and Saxena, S. C., 1996. "Heat transfer in rarefied gas at a gas-solid interface". Energy, 21, pp. 99-103.

[34] Kuehn, T., and Goldstein, R., 1976. "Correlating equations for natural convection heat transfer between horizontal circular cylinders". Int. J. Heat Mass Trans., 19(10), pp. 1127-1134.

[35] Maxwell, J. C., 1890. "The scientific papers of james clerk maxwell."

[36] Kennard, E. H., 1938. Kinetic energy of gases. Mc Graw - Hill book company.

[37] Hadj-Nacer, M., Maharjan, D., Ho, M. T., Stefanov, S. K., Graur, I., and Greiner, M., 2017. "Continuum and kinetic simulations of heat transfer trough rarefied gas in annular and planar geometries in the slip regime". J. Heat Trans., 139, pp. 042002-04010.

[38] Spurgeon, W. A., 2018. Thermal conductivities of some polymers and composites. Technical report, US Army Research Laboratory, Cambridge, MA, Feb. See also URL \\https://www.arl.army.mil/arlreports/2018/ARLTR-8298.pdf.

[39] Kittel, C., 1949. "Interpretation of the thermal conductivity of glasses". Phys. Rev., 75(6), pp. 972-974.

[40] Babcock, C. L., 1963. "Thermal conductivities of glass between $-150^{\circ} \mathrm{c}$ and $100^{\circ} \mathrm{c}$ ". Glass Technology, 4(4), Augus.

[41] Ratcliffe, E. H., 1961. "Symposium on heat transfer phenomena in glass.". J. Am. Ceram. Soc., 44(7), July.

[42] Ftouni, H., Tainoff, D., Richard, J., Lulla, K., Guidi, J., Collin, E., and Bourgeois, O., 2013. "Specific heat measurement of thin suspended sin membrane from $8 \mathrm{k}$ to $300 \mathrm{k}$ using the $3 \omega$-völklein method". Rev. Sci. Instrum., 84(9), p. 094902.

[43] Ftouni, H., Blanc, C., Tainoff, D., Fefferman, A. D., Defoort, M., Lulla, K. J., Richard, J., Collin, E., and Bourgeois, O., 2015. "Thermal conductivity of silicon nitride membranes is not sensitive to stress". Phys. Rev. B, 92(12), p. 125439.

[44] Fujii, T., Fujii, M., and Honda, T. "Theoretical and experimental studies of the free convection around a long horizontal thin wire in air.". Proceedings of the 7th International Heat Transfer Conference, Munich, Germany, 1982, 2, p. 311.

[45] Jack Hu, X., Jain, A., and K.E., G., 2008. "Investigation of the natural convection boundary condition in microfabricated structures.". International Journal of Thermal Sciences, 47, p. 820.

[46] Wang, H., Liu, J., Zhang, X., Li, T., Zhang, R., , and Wei, F., 2013. "Heat transfer between an individual carbon nanotube and gas environment in a wide knudsen number regime.". Journal of Nanomaterials, 2013, p. 3.

[47] Fujiwara, S., Zhang, X., and Fujii, M., 2001. "Short-hot-wire method for the measurement of total hemispherical emissivity of a fine fibre.". High Temperatures-High pressures, 33, p. 271.

[48] Kim, K., Chung, J., Hwang, G., Kwon, O., and Lee, J., 2011. "Quantitative measurement with scanning thermal microscope by preventing the distortion due to the heat transfer through the air.". ACS Nano, 5, p. 8700 .

[49] Shi, L., and Majumdar, A., 2002. "Thermal transport mechanisms at nanoscale point contacts.". J. Heat Transfer, 124, p. 329.

[50] Bresson, B., Brun, C., Buet, X., Chen, Y., Ciccotti, M., Gateau, J., Jasion, G., Petrovich, M., Poletti, F., Richardson, D., Sandoghchi, S., Tessier, G., Tyukodi, B., and Vandembroucq, D., 2017. "Thermal transport mechanisms at nanoscale point contacts.". Phys. Rev. Lett., 119, p. 235501.

[51] Fiorino, A., Thompson, D., Zhu, L., Song, B., Reddy, P., and Meyhofer, E., 2018. "Giant enhancement in radiative heat transfer in sub-30 $\mathrm{nm}$ gaps of plane parallel surfaces.”. Nano Lett., 18, p. 3711.

[52] Song, B., Thompson, D., Fiorino, A., Ganjeh, Y., Reddy, P., and Meyhofer, E., 2016. "Radiative heat conductances between dielectric and metallic parallel plates with nanoscale gaps.". Nature Nanotech., 11, p. 509. 